\title{
Diagnostic utility of metabolic parameters on FDG PET/CT for lymph node metastasis in patients with cN2 non-small cell lung cancer
}

Keita Nakanishi ${ }^{1}$, Shota Nakamura* ${ }^{*}$, Tomoshi Sugiyama$^{1}$, Yuka Kadomatsu ${ }^{1}$, Harushi Ueno ${ }^{1}$, Masaki Goto ${ }^{1}$ Naoki Ozeki ${ }^{1}$, Takayuki Fukui ${ }^{1}$, Shingo Iwano ${ }^{2}$ and Toyofumi Fengshi Chen-Yoshikawa ${ }^{1}$

\begin{abstract}
Background: The aim of this study was to assess the diagnostic utility of metabolic parameters on fluorine-18fluoro-2-deoxy-D-glucose-positron emission tomography (FDG-PET)/computed tomography (CT) for predicting lymph node (LN) metastasis in patients with cN2 non-small cell lung cancer (NSCLC).

Methods: We retrospectively reviewed patients who underwent surgery for CN2 NSCLC between 2007 and 2020. Those who had clinically diagnosed positive hilar and mediastinal LNs by routine CT and PET/CT imaging were investigated. To measure the metabolic parameters of LNs, the data according to maximum standardized uptake value (SUVmax), metabolic tumor volume (MTV), total lesion glycolysis (TLG), and LN-to-primary tumor ratio of SUVmax (LPR) were examined. The diagnosis of each retrieved LN was confirmed based on histopathological examination of surgical tissue specimens. Receiver operating characteristics (ROC) curves with area under the curve (AUC) calculations and multivariate analysis by logistic regression were performed.

Results: Forty-five patients with 84 clinically diagnosed positive hilar or mediastinal LNs were enrolled in the present study. Of the 84 LNs, 63 LNs were pathologically proven as positive (75\%). The SUVmax, MTV, TLG, and LPR of LN metastasis were significantly higher than those of benign nodes. In the ROC analysis, the AUC value of LPR [AUC, 0.776; 95\% confidence interval (CI), 0.640-0.913] was higher than that of LN SUVmax (AUC, 0.753; 95\% Cl, $0.626-0.880$ ) or LN TLG3.5 (AUC, $0.746 ; 95 \%$ Cl, 0.607-0.885). Using the optimal LPR cutoff value of 0.47 , the sensitivity, specificity, positive predictive value, negative predictive value, and accuracy were $84.1,66.7,88.3,58.3$, and $79.8 \%$, respectively. Multivariate analysis by logistic regression showed that LPR was an independent predictor for $\mathrm{LN}$ metastasis (odds ratio, $6.45 ; 95 \% \mathrm{Cl}, 1.785-23.301 ; P=0.004$ ). In the subgroup analysis of adenocarcinoma patients ( $n=18$; $32 \mathrm{LNs}$ ), TLG3.5 was a better predictor (AUC, 0.816; 95\% Cl, 0.639-0.985) than LPR (AUC, 0.792; 95\% $\mathrm{Cl}, 0.599-0.986$ ) or LN SUVmax (AUC, 0.792; 95\% Cl, 0.625-0.959).
\end{abstract}

Conclusions: Our findings suggest that LPR on FDG-PET is a useful predictor for LN metastasis in patients with cN2 NSCLC. TLG can be a good predictor for LN metastasis in patients with adenocarcinoma.

Keywords: Non-small cell lung cancer, FDG-PET/CT, Lymph node metastasis, Metabolic parameter

\footnotetext{
* Correspondence: shota197065@med.nagoya-u.ac.jp

'Department of Thoracic Surgery, Nagoya University Graduate School of Medicine, 65 Tsurumai-cho, Showa-ku, Nagoya 466-8550, Japan

Full list of author information is available at the end of the article
}

(c) The Author(s). 2021 Open Access This article is licensed under a Creative Commons Attribution 4.0 International License, which permits use, sharing, adaptation, distribution and reproduction in any medium or format, as long as you give appropriate credit to the original author(s) and the source, provide a link to the Creative Commons licence, and indicate if changes were made. The images or other third party material in this article are included in the article's Creative Commons. licence, unless indicated otherwise in a credit line to the material. If material is not included in the article's Creative Commons licence and your intended use is not permitted by statutory regulation or exceeds the permitted use, you will need to obtain permission directly from the copyright holder. To view a copy of this licence, visit http://creativecommons.org/licenses/by/4.0/ The Creative Commons Public Domain Dedication waiver (http://creativecommons.org/publicdomain/zero/1.0/) applies to the data made available in this article, unless otherwise stated in a credit line to the data. 


\section{Background}

Accurate lymph node (LN) staging of primary non-small cell lung cancer (NSCLC), especially in patients with clinically suspected N2, is crucial in making therapeutic strategy decisions and in determining clinical outcomes [1-3]. In recent years, after receiving the results of the Pacific trial [4], the treatment strategy for stage III unresectable locally advanced NSCLC has changed dramatically, and the importance of assessing mediastinal LN metastasis accurately has increased. In clinical practice, computed tomography (CT) and/or fluorine-18-fluoro2-deoxy-D-glucose-positron emission tomography (FDG-PET/CT) are usually performed for clinical LN staging. However, these modalities do not play a complete role in LN staging and causes diagnostic ambiguity (providing false-positive or false-negative results) in the clinical practice. Although LNs with a short-axis diameter of more than $10 \mathrm{~mm}$ on a contrast-enhanced CT are generally diagnosed as malignant, a previous meta-analysis showed a relatively low sensitivity (59\%) and specificity (78\%) [5]. In previous studies, using FDG$\mathrm{PET} / \mathrm{CT}$ in diagnosing mediastinal LNs also showed a relatively low sensitivity of $50-79 \%$ and specificity of $72-94 \%$ [6-10]. Inflammatory changes in LN due to tuberculosis or granulomatous disease can increase FDG uptake and false-positive results $[6,11]$. On the contrary, a maximum standardized uptake value (SUVmax) threshold is associated with a greater number of falsenegative findings [12, 13]. Mediastinal LN staging by endobronchial ultrasound guided-transbronchial needle aspiration (EBUS-TBNA)/mediastinoscopy was reported to be useful and was recommended when LN metastasis is suspected on imaging [14]. However, there are LNs, including those in stations \#5 or \#6, which are difficult to evaluate with EBUS-TBNA. Furthermore, there are possible complications, such as bleeding and mediastinitis [15]. Hence, a more accurate and less invasive diagnosis of $\mathrm{LN}$ involvement is required in patients with $\mathrm{cN} 2$ NSCLC.

In recent years, the usefulness of metabolic parameters, including metabolic tumor volume (MTV) and total lesion glycolysis (TLG) has been investigated. Several studies reported that MTV and TLG in the primary tumor of lung cancer, which provides information on tumor activity and volume, could predict the pathological tumor invasive size [16], LN status [17, 18], and prognosis [19-22] more accurately than SUVmax. Furthermore, the LN-to-primary tumor ratio of SUVmax (LPR) was also assessed to determine whether this parameter can be a good predictor for LN metastasis in NSCLC patients [13, 23]. However, only a few studies examined the diagnostic utility of these metabolic parameters and focused not on the primary tumor but on LNs for LN staging of primary lung cancer. Therefore, we aimed to investigate the role of metabolic parameters on FDG-PET/CT for LN metastasis in patients with $\mathrm{cN} 2$ NSCLC.

\section{Methods \\ Patient selection}

Overall, 2407 patients underwent surgery for primary NSCLC at Nagoya University Hospital between January 2007 and March 2020. Among them, 113 patients who underwent PET before surgery and upfront lung resection for $\mathrm{cN} 2 \mathrm{NSCLC}$ were retrospectively identified. As illustrated in Fig. 1, the following patients were excluded: those who received induction therapy $(n=36)$, who underwent PET in other institution $(n=26)$, with uncontrolled diabetes mellitus $(n=4)$, and with synchronous malignancy $(n=2)$. The present study was approved by the institutional review board of our institute (approval No. 2020-0375), and informed consent was obtained from each patient for the use of clinical data in various investigations.

\section{Data collection}

Institutional databases and medical records of each patient were retrospectively reviewed to determine age, sex, tumor location, tumor size, clinical tumor-nodemetastasis staging, histology, and surgical procedure. Staging was determined based on the seventh edition of the tumor, node, and metastasis classification for lung cancer [24]. LN stations were determined based on the International Association for the Study of Lung Cancer LN map [25]. At our institution, prior to treatment, LNs that were clinically diagnosed as positive were defined as measuring $>1.0 \mathrm{~cm}$ in the short-axis diameter on the CT image [5] with a SUVmax value of $>2.5$ on PET/CT [26]. Although EBUS-TBNA was not mandatory in the present study, preoperative confirmation of $\mathrm{N} 2$ by EBUS-TBNA was performed in 10 patients (22\%). The diagnosis of the retrieved LN was confirmed based on histopathological examination of surgical tissue specimens.

\section{FDG-PET/CT scan protocol and image evaluation}

FDG-PET/CT was performed as previously described [16]. PET/CT was carried out using a Biograph 16 scanner (Siemens Healthcare) within 31 days prior to surgery. The blood glucose levels were measured immediately before FDG injection. Patients with a blood glucose level greater than $150 \mathrm{mg} / \mathrm{dL}$ were not allowed to undergo PET/CT. The FDG dose was determined based on the body weight, using either 3.7 (for patients weighing $<60$ $\mathrm{kg}$ ) or 4.07 (for those weighing $\geq 60 \mathrm{~kg}$ ) MBq/ $\mathrm{kg}$. Breathholding and respiratory gating techniques were not applied. To measure the metabolic parameters, preexisting PET data were reanalyzed using the MM oncology 


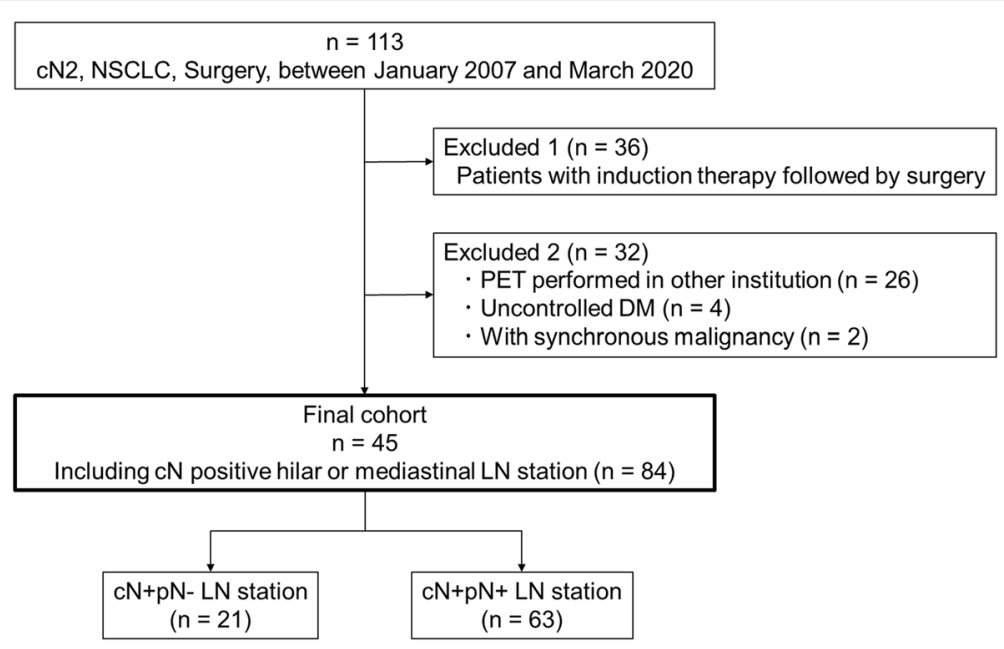

Fig. 1 Patient selection. NSCLC, non-small cell lung cancer; PET, positron emission tomography; DM, diabetes mellitus; LN, lymph node

software on a syngo.via workstation (Siemens Healthcare). All PET images were retrospectively evaluated by three investigators (K.N., S.N., and S.I.), including two certified thoracic surgeons and one radiologist specializing in chest radiology and nuclear medicine. The radiologist set the three-dimensional volume of interest to sufficiently cover the primary tumor and LNs clinically diagnosed as positive on a PET/CT images, and the data according to SUVmax, MTV, and TLG were automatically extracted (Fig. 2). The SUVmax was defined as the maximum value of the volume of interest. The LPR was calculated as LN SUVmax divided by primary tumor SUVmax. The MTV was defined as the tumor volume over a threshold value of SUV, which varied from 2.5 to 5.0. The TLG was obtained by multiplying MTV with its
SUVmean, which varied from 2.5 to 5.0. All MTV and TLG threshold values from 2.5 to 5.0 in 0.5 increments were investigated during the study period. However, MTV3.5 and TLG3.5 were adopted in the analysis phase since these values were the most sensitive threshold values.

\section{Statistical analysis}

The differences between groups were compared using the Mann-Whitney U test for continuous variables. Receiver operating characteristics (ROC) curves with area under the curve (AUC) calculations were performed to determine whether SUVmax, MTV, TLG, and LPR can predict nodal status and to determine the optimal cutoff values by Youden's Index. A multivariate logistic
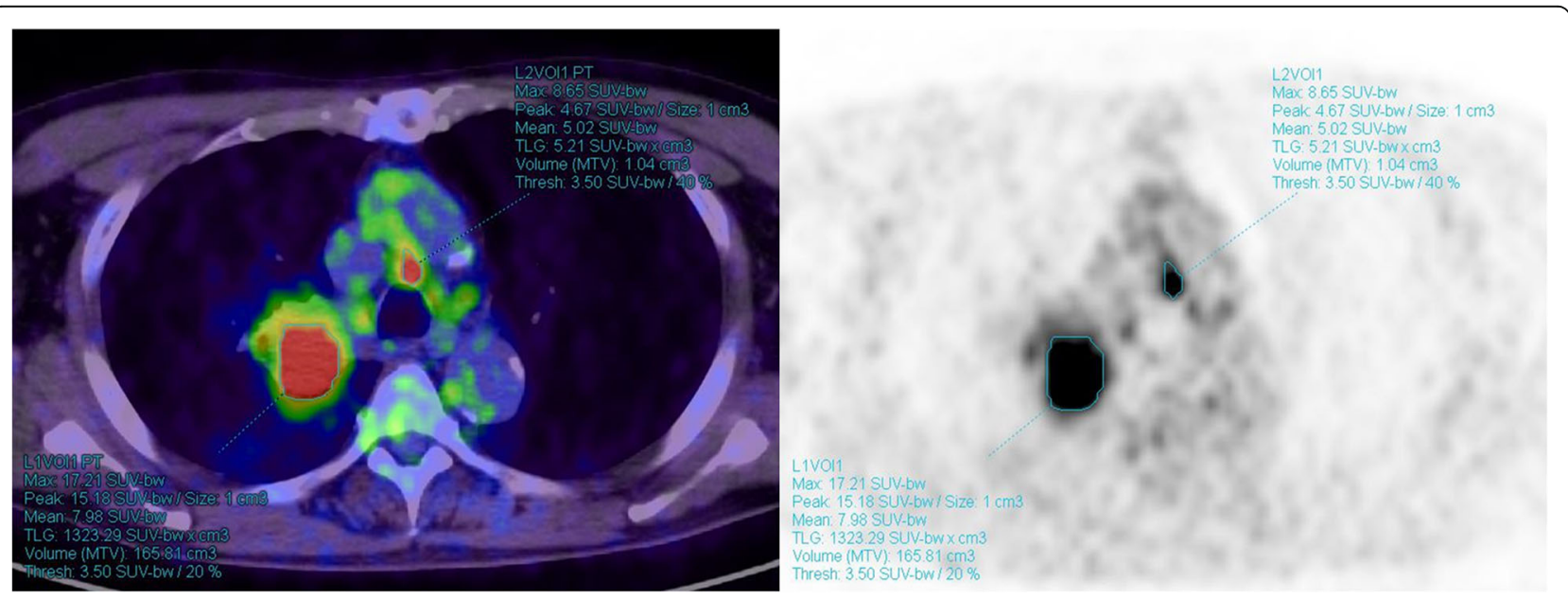

Fig. 2 Representative example of the measurement of metabolic parameters on FDG-PET/CT for primary tumor and LNs. Primary tumor with an SUVmax of 17.21, MTV3.5 of $165.81 \mathrm{~cm}^{3}$, and TLG3.5 of 1323.29; \#4R LN with an SUVmax of 8.65, MTV3.5 of $1.04 \mathrm{~cm}^{3}$, TLG3.5 of 5.21 , and LPR of 0.5. In the pathological report, the LN was diagnosed as positive. FDG-PET/CT, fluorine-18-fluoro-2-deoxy-D-glucose-positron emission tomography; LN, lymph node; SUVmax, maximum standardized uptake value; MTV, metabolic tumor volume; TLG, total lesion glycolysis; LPR, lymph node-to-primary tumor ratio of SUVmax 
regression analysis that considered significant risk factors from ROC analysis, except for the factor with multicollinearity, was performed. For all analyses, $P$-values of $<0.05$ were considered statistically significant. All statistical analyses were performed using the SPSS Statistics 25 software (IBM Corporation, Armonk, NY).

\section{Results}

Forty-five patients with 84 clinically diagnosed positive hilar or mediastinal LNs were enrolled in the present study. The clinicopathological characteristics are summarized in Table 1. All cohorts comprised 39 men (87\%) and six women, with a median age of 70 years (40-79).

Table 1 Baseline characteristics

\begin{tabular}{|c|c|}
\hline Characteristics & $n=45(\%)$ \\
\hline Age, median (range) & $70(40-79)$ \\
\hline Sex, male & $39(87)$ \\
\hline \multicolumn{2}{|l|}{ Tumor location } \\
\hline RUL & $16(36)$ \\
\hline RML & $1(2)$ \\
\hline RLL & $12(27)$ \\
\hline LUL & $11(24)$ \\
\hline LLL & $3(7)$ \\
\hline RMLL & $2(4)$ \\
\hline Tumor size, mm, median (range) & $35(9-106)$ \\
\hline \multicolumn{2}{|l|}{ Clinical T factor } \\
\hline $\mathrm{T} 1$ & $16(35)$ \\
\hline $\mathrm{T} 2$ & $17(38)$ \\
\hline $\mathrm{T} 3$ & $8(18)$ \\
\hline $\mathrm{T} 4$ & $4(9)$ \\
\hline \multicolumn{2}{|l|}{ Histology } \\
\hline Adenocarcinoma & $18(40)$ \\
\hline Squamous cell carcinoma & $18(40)$ \\
\hline Others $^{a}$ & $9(20)$ \\
\hline \multicolumn{2}{|l|}{ Procedure of operation } \\
\hline Lobectomy & $34(76)$ \\
\hline Bi-lobectomy & $7(16)$ \\
\hline Pneumonectomy & $2(4)$ \\
\hline No resection (only LN sampling) & $2(4)$ \\
\hline \multicolumn{2}{|l|}{ Lymph node dissection } \\
\hline Only LN sampling & $2(4)$ \\
\hline ND2a-1 & $7(16)$ \\
\hline ND2a-2 & $34(76)$ \\
\hline $\mathrm{ND} 2 \mathrm{~b}$ & $2(4)$ \\
\hline
\end{tabular}

$R U L$ right upper lobe, $R M L$ right middle lobe, $R L L$ right lower lobe, $L U L$ left upper lobe, $L L L$ left lower lobe, $R M L L$ right middle and lower lobe, $L N$ lymph node, ND node dissection

${ }^{a}$ Including adenosquamous cell carcinoma, large cell carcinoma and neuroendocrine tumors
The median tumor size was $35 \mathrm{~mm}(9-106 \mathrm{~mm})$, and the clinical $\mathrm{T}$ factor was mainly $\mathrm{T} 1-\mathrm{T} 2$ (73\%). The primary histology was adenocarcinoma in 18 patients (40\%), squamous cell carcinoma in 18 patients (40\%), and others including adenosquamous cell carcinoma or large cell carcinoma and neuroendocrine tumors in nine patients (20\%). In the present study, lobectomy was mainly performed (76\%), and systemic LN dissection of more than ND2a-2 was performed on 36 patients (80\%).

Of the 84 hilar or mediastinal LNs clinically diagnosed as positive, 63 were pathologically proven as positive (75\%), whereas 21 were pathologically proven as negative (25\%). The FDG-PET/CT parameters for negative and positive LNs are presented in Table 2. The SUVmax, MTV, TLG, and LPR of metastatic LNs were higher than those of benign nodes. No significant difference was observed in the primary tumor SUVmax. In the ROC analysis, the AUC value of LPR [AUC, 0.776; 95\% confidence interval (CI), 0.640-0.913] was higher than that of LN SUVmax (AUC, 0.753; 95\% CI, 0.626-0.880) or LN TLG3.5 (AUC, 0.746; 95\% CI, 0.607-0.885) (Fig. 3a). MTV had the lowest AUC value compared with the above parameters (not shown in Fig. 3a). Using the optimal LPR cutoff value of 0.47 , the sensitivity, specificity, positive predictive value, negative predictive value, and accuracy were 84.1, 66.7, 88.3, 58.3, and $79.8 \%$, respectively. On the contrary, the LN SUVmax (cutoff value, 4.15) and LN TLG3.5 (cutoff value, 1.26) showed sensitivity values of 79.4 and $81.0 \%$, specificity values of 76.2 and $76.2 \%$, positive predictive values of 90.9 and $91.1 \%$, negative predictive values of 55.2 and $57.1 \%$, and accuracies of 78.6 and $79.8 \%$, respectively. The results of the multivariate analysis of predictors associated with LN metastasis by logistic regression are shown in Table 3. LPR was the independent predictor for $\mathrm{LN}$ metastasis (odds ratio, 6.45; 95\% CI, 1.78523.301; $P=0.004)$.

A subgroup analysis based on the histology of primary tumor was performed. In the subgroup analysis of patients with adenocarcinoma $(n=18 ; 32 \mathrm{LNs})$, the SUVmax, MTV, TLG, and LPR of metastatic LNs were higher than those of benign nodes, but there was no significant difference in primary tumor SUVmax (Supplementary Table S1). In the subgroup analysis of patients with squamous cell carcinoma ( $n=18 ; 34$ LNs), the SUVmax and LPR of metastatic LNs were higher than those of benign nodes, but there were no significant differences in primary tumor SUVmax, MTV and TLG of LNs (Supplementary Table S2). In the ROC analysis based on adenocarcinoma, TLG3.5 was a better predictor (AUC, 0.816; 95\% CI, 0.639-0.985) than LPR (AUC, 0.792; 95\% CI, 0.599-0.986) or LN SUVmax (AUC, 0.792; 95\% CI, 0.625-0.959) (Fig. 3b). Using the optimal TLG3.5 cutoff value of 1.26 , the sensitivity, 
Table 2 FDG-PET/CT parameters for negative and positive LNs

\begin{tabular}{|c|c|c|c|}
\hline Characteristics & Negative LNs $(n=21)$ & Positive LNs $(n=63)$ & $P$ value \\
\hline \multicolumn{4}{|l|}{ LN location } \\
\hline \multicolumn{4}{|l|}{ N2 nodes } \\
\hline \multicolumn{4}{|c|}{ Superior mediastinal nodes } \\
\hline$\# 2 R$ & 2 & 0 & \\
\hline$\# 3 p$ & 1 & 1 & \\
\hline$\# 4 \mathrm{R}$ & 6 & 14 & \\
\hline$\# 4 \mathrm{~L}$ & 0 & 3 & \\
\hline \multicolumn{4}{|l|}{ Aortic nodes } \\
\hline \#5-\#6 & 0 & 10 & \\
\hline \multicolumn{4}{|c|}{ Inferior mediastinal nodes } \\
\hline \#7 & 4 & 15 & \\
\hline \#8-\#9 & 0 & 2 & \\
\hline \multicolumn{4}{|l|}{ N1 nodes } \\
\hline$\# 10$ & 2 & 3 & \\
\hline$\# 11$ & 4 & 9 & \\
\hline$\# 12$ & 2 & 6 & \\
\hline Primary tumor SUVmax & $10.66(2.55-17.91)$ & $9.04(1.66-18.67)$ & 0.09 \\
\hline LN SUVmax & $3.54(2.52-11.20)$ & $5.77(2.81-15.54)$ & 0.001 \\
\hline LN MTV3.5 & $0.01(0-13.36)$ & $1.44(0-22.68)$ & 0.001 \\
\hline LN TLG3.5 & $0.05(0-71.25)$ & $6.34(0-162.78)$ & 0.001 \\
\hline LPR & $0.37(0.15-1.76)$ & $0.81(0.26-2.26)$ & $<0.001$ \\
\hline
\end{tabular}

FDG-PET/CT fluorine-18-fluoro-2-deoxy-D-glucose positron emission tomography, LN lymph node, SUVmax maximum standardized uptake value, MTV metabolic tumor volume, $T L G$ total lesion glycolysis, $L P R$ lymph node-to-primary tumor ratio of SUVmax

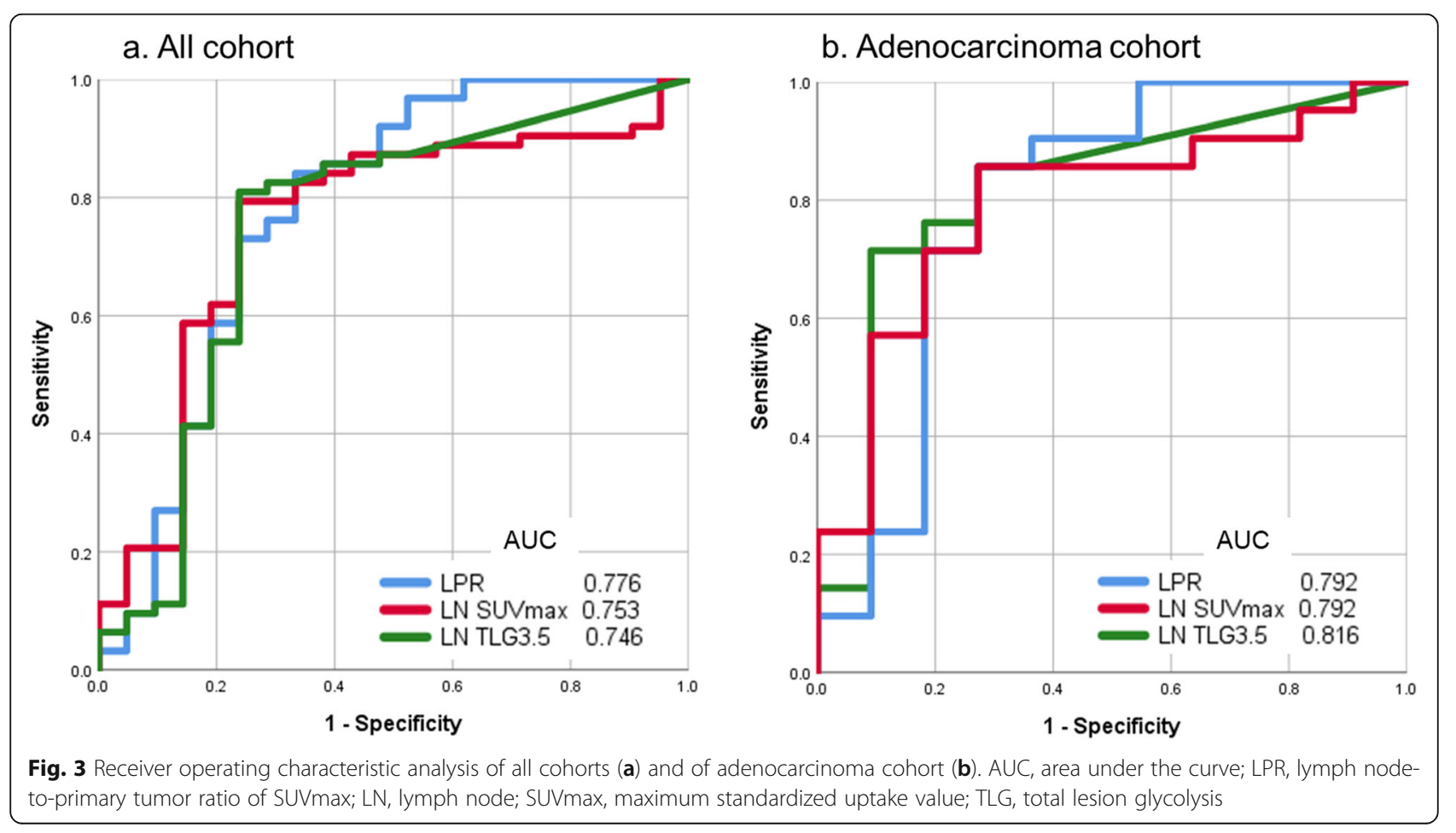


Table 3 Multivariate analysis of predictors associated with LN metastasis by logistic regression

\begin{tabular}{llll}
\hline Characteristics & OR & $\mathbf{9 5 \% C l}$ & $\boldsymbol{P}$ value \\
\hline LN SUVmax & 1.037 & $0.053-20.207$ & 0.98 \\
LN TLG3.5 & 8.727 & $0.459-166.02$ & 0.15 \\
LPR & 6.450 & $1.785-23.301$ & 0.004
\end{tabular}

LN lymph node, SUVmax maximum standardized uptake value, $T L G$ total lesion glycolysis, $L P R$ lymph node-to-primary tumor ratio of SUVmax, OR odds ratio, $\mathrm{Cl}$ confidence interval

specificity, positive predictive value, negative predictive value, and accuracy were 71.4, 90.9, 93.8, 62.5, and $78.1 \%$, respectively. In the ROC analysis based on squamous cell carcinoma, LPR was a better predictor (AUC, 0.831; 95\% CI, 0.642-1.000) than LN SUVmax (AUC, 0.804; 95\% CI, 0.593-1.000) or TLG3.5 (AUC, 0.701; 95\% CI, 0.436-0.966). On the other hand, there was no predictor in other histological group. A subgroup analysis based on the LN location was performed. All LNs were assigned to the following two groups: N2 nodes $(n=58)$ and N1 nodes $(n=26)$; PET parameters were compared between these groups. The LN SUVmax, MTV, and TLG of N1 nodes were significantly higher than those of N2 nodes, but there was no significant difference in the LPR between the two groups (Supplementary Table S3). In the ROC analysis, the AUC value of LPR was higher than that of the LN SUVmax or TLG3.5 in the $\mathrm{N} 2$ nodes as well as in the entire cohort (AUC, 0.908; 95\% CI, 0.821-0.995) (Supplementary Fig. S1). On the other hand, there was no significant predictor in the N1 nodes.

\section{Discussion}

The present study suggested that LPR on FDG-PET before surgery, and not SUVmax or TLG, is the best predictor of LN metastasis in patients with cN2 NSCLC. TLG can be a good predictor for LN metastasis in patients with adenocarcinoma. To the best of our knowledge, this is the first study to investigate metabolic parameters such as MTV or TLG for the diagnosis of LN metastasis. This study evaluated the presence of LN metastasis through surgical pathology, including \#5 or \#6 LN station, which was difficult to evaluate with EBUS-TBNA.

In patients with NSCLC, accurate preoperative LN evaluation is required, especially in those with clinically suspected mediastinal LN metastasis. This is because the status of mediastinum LNs determines the patient's treatment strategies or modalities. The present study demonstrated that the sensitivity and specificity for LN metastasis were 84.1 and $66.7 \%$ using LPR, which could reduce the false-negative findings due to its high sensitivity. In other words, LPR was useful for the exclusionary diagnosis of LN metastasis. These results might highlight the issue of false-positive test results, which resulted from a small volume of benign LN being present or relatively high metabolic activity or both. Several previous studies reported the usefulness of LPR when assessing LN status $[13,23]$. In a series of 44 patients with 92 mediastinal LNs with a SUVmax of 2.5-4.0, Moloney et al. reported that the LPR of 0.3 was the optimal cutoff value for predicting malignancy (sensitivity, 91\%; specificity, 71\%) [13]. In a series of 172 patients with 504 PETpositive LNs, Mattes and colleagues found that a cutoff value of 0.28 was optimal (sensitivity, 93\%; specificity, 87\%) [23]. Their optimal cutoff values were somewhat lower than that of the present study, which was 0.47 . This was most likely due to study population differences. For example, the former study excluded LNs with a SUVmax of more than 4.0, while the latter study included LNs with a SUV max of less than 2.5. Although there were differences in the inclusion criteria, background, and rate of LN malignancy, these results, according to LPR with a high sensitivity and a relatively low specificity, supported our results.

On the contrary, using LPR reduced the specificity and increased the number of false-positive cases. This result might be associated with the concurrent infectious disease such as obstructive pneumonia and granulomatous inflammation owing to the relatively high prevalence of tuberculosis in our ethnic background. According to predictors for LN metastasis using the SUV threshold, previous studies showed high specificity but relatively low sensitivity [6-10]. Darling and colleagues reported the accuracy of PET-CT in 149 patients who underwent mediastinoscopy and/or thoracotomy for mediastinal staging [9]. Although their study had PET-CT sensitivity of $70 \%$ and specificity of $94 \%$, they highlighted the risk of missing the chance of surgery because of the relatively high number of false-positive findings. There are limitations in comparing the sensitivities, specificities, and other parameters between studies due to the heterogeneity of the prevalence of malignancies, ethnic background, tumor histology, and cutoff values. However, including the LPR into the diagnostic approach could increase the accuracy for predicting LN metastasis. Indeed, in a subgroup analysis, the AUC value of the LPR was higher than that of the LN SUVmax or TLG3.5, especially in N2 nodes. We considered that tumor volume had an effect on hilar LNs, but SUV uptake rather than tumor volume may have had a stronger effect on mediastinal LNs. Therefore, LPR was the best predictor of LN metastasis, especially in $\mathrm{N} 2$ nodes.

In recent years, the usefulness of metabolic parameters, including MTV and TLG, has been investigated. Several studies could predict the LN status according to MTV and TLG in the primary tumor of lung cancer [17, 18]. However, no studies have investigated metabolic 
parameters of LN itself, and whether these parameters were useful remains unclear. We also investigated the usefulness of metabolic parameters, and revealed the association between those parameters and pathological invasive size in patients with adenocarcinoma [16]. Initially, we assumed that these metabolic parameters would have a more diagnostic value in predicting $\mathrm{LN}$, as well as the primary tumor, but the results were contrary to that hypothesis. This might be because, unlike the primary tumor, the metabolic activity in the $\mathrm{LN}$ was lower and the difference in LN volume was less apparent. In cases of adenocarcinoma, TLG had a higher diagnostic value than LPR. Although the mechanism was unknown, tumor activity or glucose transporter family expression might be different at the metastatic site depending on the tissue type [27]. Hence, further studies are warranted to clarify this mechanism.

The present study has several limitations. First, our retrospective study revealed a single-center investigation conducted in individuals with the same ethnic background and geographical region. Therefore, the insufficiency of data and external validity are potential problems. Second, there was one limitation to confirm that the LN evaluated on imaging was the same LN diagnosed pathologically in any study of this nature. Third, our study had a small sample size. PET scans performed at other institutions were excluded to ensure uniform FDG-PET/CT imaging accuracy. A further prospective multicenter study is needed to assess not only the diagnostic ability but also the effect of metabolic parameters on making treatment decisions.

\section{Conclusions}

Our findings suggest that LPR on FDG-PET is a useful predictor for LN metastasis in patients with $\mathrm{CN} 2 \mathrm{NSCL}$ C. In addition, TLG can be a good predictor for LN metastasis in patients with adenocarcinoma. Further prospective, multi-institutional study is needed to validate the outcomes of the present study and to assess the effect of metabolic parameters on the therapeutic strategy.

\begin{abstract}
Abbreviations
LN: Lymph node; NSCLC: Non-small cell lung cancer; CT: Computed tomography; FDG-PET: Fluorine-18-fluoro-2-deoxy-D-glucose-positron emission tomography; SUV: Standardized uptake value; EBUSTBNA: Endobronchial ultrasound guided-transbronchial needle aspiration; MTV: Metabolic tumor volume; TLG: Tumor lesion glycolysis; LPR: Lymph-toprimary tumor ratio of SUVmax; ROC: Receiver operating characteristics; AUC: Area under the curve
\end{abstract}

\section{Supplementary Information}

The online version contains supplementary material available at https://doi. org/10.1186/s12885-021-08688-6

Additional file 1 : Supplementary Table S1. FDG-PET/CT parameters for negative and positive LNs in adenocarcinoma. FDG-PET/CT, fluorine-
18-fluoro-2-deoxy-D-glucose positron emission tomography/ computed tomography; LN, lymph node; SUVmax, maximum standardized uptake value; MTV, metabolic tumor volume; TLG, total lesion glycolysis; LPR, lymph node-to-primary tumor ratio of SUVmax. Supplementary Table S2. FDG-PET/CT parameters for negative and positive LNs in squamous cell carcinoma. FDG-PET/CT, fluorine-18-fluoro-2-deoxy-D-glucose positron emission tomography/ computed tomography; LN, lymph node; SUVmax, maximum standardized uptake value; MTV, metabolic tumor volume; TLG, total lesion glycolysis; LPR, lymph node-to-primary tumor ratio of SUVmax. Supplementary Table S3. FDG-PET/CT parameters according to LN location. FDG-PET/CT, fluorine-18-fluoro-2-deoxy-D-glucose positron emission tomography/ computed tomography; LN, lymph node: SUVmax, maximum standardized uptake value; MTV, metabolic tumor volume; TLG, total lesion glycolysis; LPR, lymph node-to-primary tumor ratio of SUVmax.

Additional file 2 : Supplementary Figure S1. Receiver operating characteristic analysis of N2 nodes (Figure S1a) and of N1 nodes (Figure $\mathrm{S} 1 \mathrm{~b}) . \mathrm{AUC}$, area under the curve; LPR, lymph node-to-primary tumor ratio of SUVmax; LN, lymph node; SUVmax, maximum standardized uptake value; $T L G$, total lesion glycolysis.

\section{Acknowledgements}

The authors thank Dr. Katsuhiko Kato of the Department of Radiological and Medical Laboratory Sciences of Nagoya University Graduate School of Medicine for giving motivation for research. The authors also thank Enago (www.enago.jp) for English language editing.

\section{Authors' contributions}

$\mathrm{KN}$ contributed to data collection and manuscript writing. $\mathrm{KN}$, SN, and $\mathrm{SI}$ were major contributors in collecting, analyzing, and interpreting the patient data. TS, YK, HU, MG, NO, and TF contributed to data collection. TFC-Y contributed to the design of this study. All authors contributed to the draft and revisions of this manuscript. All authors read and approved the final manuscript.

\section{Funding}

Not applicable.

\section{Availability of data and materials}

The datasets used and/or analysed during the current study available from the corresponding author on reasonable request.

\section{Declarations}

Ethics approval and consent to participate

All procedures performed on our participants were in accordance with the ethical standards of the institution and/ or the national research committee and with the 1964 Helsinki declaration and its later amendments or comparable ethical standards. This retrospective study was approved by the institutional review board of Nagoya University Graduate School of Medicine (approval No. 2020-0375), and informed consent was obtained from each patient for the use of clinical data in various investigations.

Consent for publication

Not applicable.

\section{Competing interests}

The authors declare that they have no competing interests.

\section{Author details}

'Department of Thoracic Surgery, Nagoya University Graduate School of Medicine, 65 Tsurumai-cho, Showa-ku, Nagoya 466-8550, Japan. ${ }^{2}$ Department of Radiology, Nagoya University Graduate School of Medicine, Nagoya, Japan. 
Received: 5 April 2021 Accepted: 16 August 2021

Published online: 02 September 2021

\section{References}

1. Rami-Porta R, Bolejack V, Crowley J, Ball D, Kim J, Lyons G, et al. The IASLC lung cancer staging project: proposals for the revisions of the $t$ descriptors in the forthcoming eighth edition of the TNM classification for lung cancer. J Thorac Oncol. 2015;10(7):990-1003. https://doi.org/10.1097/JTO. 0000000000000559.

2. Asamura H, Chansky K, Crowley J, Goldstraw P, Rusch WW, Vansteenkiste JF, et al. The international association for the study of lung cancer lung cancer staging project: proposals for the revision of the $n$ descriptors in the forthcoming 8th edition of the TNM classification for lung cancer. J Thorac Oncol. 2015;10(12):1675-84. https://doi.org/10.1097/JTO.0000000000000678.

3. Eberhardt WE, Mitchell A, Crowley J, Kondo H, Kim YT, Turrisi A 3rd, et al. The IASLC lung cancer staging project: proposals for the revision of the $m$ descriptors in the forthcoming eighth edition of the TNM classification of lung cancer. J Thorac Oncol. 2015;10(11):1515-22. https://doi.org/10.1097/ JTO.0000000000000673.

4. Antonia SJ, Villegas A, Daniel D, Vicente D, Murakami S, Hui R, et al. Overall survival with durvalumab after chemoradiotherapy in stage III NSCLC. N Engl J Med. 2018;379(24):2342-50. https://doi.org/10.1056/NEJMoa1809697.

5. Birim O, Kappetein AP, Stijnen T, Bogers AJ. Meta-analysis of positron emission tomographic and computed tomographic imaging in detecting mediastinal lymph node metastases in nonsmall cell lung cancer. Ann Thorac Surg. 2005;79(1):375-82. https://doi.org/10.1016/j.a thoracsur.2004.06.041.

6. Lee JW, Kim BS, Lee DS, Chung JK, Lee MC, Kim S, et al. 18F-FDG PET/CT in mediastinal lymph node staging of non-small-cell lung cancer in a tuberculosis-endemic country: consideration of lymph node calcification and distribution pattern to improve specificity. Eur I Nucl Med Mol Imaging. 2009;36(11):1794-802. https://doi.org/10.1007/s00259-009-1155-4.

7. Lu P, Sun Y, Sun Y, Yu L. The role of (18) F-FDG PET/CT for evaluation of metastatic mediastinal lymph nodes in patients with lung squamous-cell carcinoma or adenocarcinoma. Lung Cancer. 2014;85(1):53-8. https://doi. org/10.1016/j.lungcan.2014.04.004.

8. Li X, Zhang H, Xing L, Ma H, Xie P, Zhang L, et al. Mediastinal lymph nodes staging by 18F-FDG PET/CT for early stage non-small cell lung cancer: a multicenter study. Radiother Oncol. 2012;102(2):246-50. https://doi.org/10.1 016/j.radonc.2011.10.016.

9. Darling GE, Maziak DE, Inculet RI, Gulenchyn KY, Driedger AA, Ung YC, et al. Positron emission tomography-computed tomography compared with invasive mediastinal staging in non-small cell lung cancer: results of mediastinal staging in the early lung positron emission tomography trial. Thorac Oncol. 2011;6(8):1367-72. https://doi.org/10.1097/JTO.0b013e31822 Oc912.

10. Lee AY, Choi SJ, Jung KP, Park JS, Lee SM, Bae SK. Characteristics of metastatic mediastinal lymph nodes of non-small cell lung cancer on preoperative F-18 FDG PET/CT. Nucl Med Mol Imaging. 2014;48(1):41-6. https://doi.org/10.1007/s13139-013-0244-2.

11. Kwon SY, Min JJ, Song HC, Choi C, Na KJ, Bom HS. Impact of lymphoid follicles and histiocytes on the false-positive FDG uptake of lymph nodes in non-small cell lung cancer. Nucl Med Mol Imaging. 2011;45(3):185-91. https://doi.org/10.1007/s13139-011-0085-9.

12. Bryant AS, Cerfolio RJ, Klemm KM, Ojha B. Maximum standard uptake value of mediastinal lymph nodes on integrated FDG-PET-CT predicts pathology in patients with non-small cell lung cancer. Ann Thorac Surg. 2006;82(2): 417-22. https://doi.org/10.1016/j.athoracsur.2005.12.047.

13. Moloney F, Ryan D, McCarthy L, McCarthy J, Burke L, Henry MT, et al. Increasing the accuracy of 18F-FDG PET/CT interpretation of "mildly positive" mediastinal nodes in the staging of non-small cell lung cancer. Eur J Radiol. 2014;83(5):843-7. https://doi.org/10.1016/j.ejrad.2014.01.016.

14. Bousema JE, van Dorp M, Hoeijmakers F, Huijbregts IA, Barlo NP, Bootsma GP, et al. Guideline adherence of mediastinal staging of non-small cell lung cancer: a multicentre retrospective analysis. Lung Cancer. 2019;134:52-8. https://doi.org/10.1016/j.lungcan.2019.05.031.

15. Asano F, Aoe M, Ohsaki Y, Okada Y, Sasada S, Sato S, et al. Complications associated with endobronchial ultrasound-guided transbronchial needle aspiration: a nationwide survey by the Japan Society for Respiratory Endoscopy. Respir Res. 2013;14(1):50. https://doi. org/10.1186/1465-9921-14-50.
16. Iwano S, Ito S, Kamiya S, Ito R, Kato K, Naganawa S. Utility of metabolic parameters on FDG PET/CT in the classification of early-stage lung adenocarcinoma: prediction of pathological invasive size. Clin Nucl Med. 2019:44(7):560-5. https://doi.org/10.1097/RLU.0000000000002591.

17. Kim DH, Song BI, Hong CM, Jeong SY, Lee SW, Lee J, et al. Metabolic parameters using ${ }^{18}$ F-FDG PET/CT correlate with occult lymph node metastasis in squamous cell lung carcinoma. Eur J Nucl Med Mol Imaging 2014:41(11):2051-7. https://doi.org/10.1007/s00259-014-2831-6.

18. Ouyang ML, Tang K, Xu MM, Lin J, Li TC, Zheng XW. Prediction of occult lymph node metastasis using tumor-to-blood standardized uptake ratio and metabolic parameters in clinical NO lung adenocarcinoma. Clin Nucl Med. 2018;43(10):715-20. https://doi.org/10.1097/RLU.0000000000002229.

19. Satoh Y, Onishi H, Nambu A, Araki T. Volume-based parameters measured by using FDG PET/CT in patients with stage I NSCLC treated with stereotactic body radiation therapy: prognostic value. Radiology. 2014; 270(1):275-81. https://doi.org/10.1148/radiol.13130652.

20. Wang $X Y$, Zhao YF, Liu Y, Yang YK, Wu N. rognostic value of metabolic variables of [18F] FDG PET/CT in surgically resected stage I lung adenocarcinoma. Medicine (Baltimore). 2017;96:e7941.

21. Sharma A, Mohan A, Bhalla AS, Sharma MC, Vishnubhatla S, Das CJ, et al. Role of various metabolic parameters derived from baseline 18F-FDG PET/ $\mathrm{CT}$ as prognostic markers in non-small cell lung cancer patients undergoing platinum-based chemotherapy. Clin Nucl Med. 2018;43(1):e8-17. https://doi. org/10.1097/RLU.0000000000001886.

22. Jin F, Qu B, Fu Z, Zhang Y, Han A, Kong L, et al. Prognostic value of metabolic parameters of metastatic lymph nodes on 18 F-FDG PET/CT in patients with limited-stage small-cell lung cancer with lymph node involvement. Clin Lung cancer. 2018;19(1):e101-8. https://doi.org/10.1016/j. cllc.2017.06.006

23. Mattes MD, Moshchinsky AB, Ahsanuddin S, Rizk NP, Foster A, Wu AJ, et al. Ratio of lymph node to primary tumor SUV on PET/CT accurately predicts nodal malignancy in non-small-cell lung cancer. Clin Lung Cancer. 2015: 16(6):e253-8. https://doi.org/10.1016/j.cllc.2015.06.001.

24. Rami-Porta R, Crowley JJ, Goldstraw P. The revised TNM staging system for lung cancer. Ann Thorac Cardiovasc Surg. 2009;15(1):4-9.

25. Rusch WW, Asamura H, Watanabe H, Giroux DJ, Rami-Porta R, Goldstraw P. The IASLC lung cancer staging project: a proposal for a new international lymph node map in the forthcoming seventh edition of the TNM classification for lung cancer. J Thorac Oncol. 2009;4(5):568-77. https://doi. org/10.1097/JTO.0b013e3181a0d82e.

26. Hellwig D, Graeter TP, Ukena D, Groeschel A, Sybrecht GW, Schaefers HJ, et al. 18F-FDG PET for mediastinal staging of lung cancer: which SUV threshold makes sense? J Nucl Med. 2007;48(11):1761-6. https://doi.org/10.2 967/jnumed.107.044362.

27. Taira N, Atsumi E, Nakachi S, Takamatsu R, Yohena T, Kawasaki H, et al. Comparison of GLUT-1, SGLT-1, and SGLT-2 expression in false-negative and true-positive lymph nodes during the 18 F-FDG PET/CT mediastinal nodal staging of non-small cell lung cancer. Lung Cancer. 2018;123:30-5. https:// doi.org/10.1016/j.lungcan.2018.06.004.

\section{Publisher's Note}

Springer Nature remains neutral with regard to jurisdictional claims in published maps and institutional affiliations.
Ready to submit your research? Choose BMC and benefit from:
- fast, convenient online submission
- thorough peer review by experienced researchers in your field
- rapid publication on acceptance
- support for research data, including large and complex data types
- gold Open Access which fosters wider collaboration and increased citations
- maximum visibility for your research: over $100 \mathrm{M}$ website views per year
At BMC, research is always in progress.
Learn more biomedcentral.com/submissions 